\title{
Editorial: Photonic integration and photonics-electronics convergence on silicon platform
}

\author{
Koji Yamada* \\ National Institute of Advanced Industrial Science and Technology, Tsukuba, Japan
}

Keywords: silicon photonics, photonic integration, additional waveguide system, III-V semiconductors, germanium-based emitter, wafer bonding, telecommunications applications, bio-chemical applications

Silicon-based photonics technology, which is based on the same paradigm of silicon (Si) electronics technology, promises to provide us with a compact photonic integration platform with high integration density, mass manufacturing, and excellent cost performance. This technology has been used to develop various photonic devices based on silicon, such as waveguides, filters, and modulators. In addition, germanium $(\mathrm{Ge})$ photodetectors have been built on a silicon-based photonic platform. These photonic devices have already been monolithically integrated on silicon chips. Moreover, photonics-electronics convergence based on silicon photonics is now being pursued. These emerging compact photonics-electronics convergent modules have the potential to be used in the fabrication of energy-efficient cost-effective systems for various applications, such as communications, information processing, and sensing.

The last decade first saw the development of Si-based photonic technologies for communication applications, and commercial products are now available for short-range data communications. For medium-/long-range telecommunication applications, in which stringent technical standards are applied to guarantee long-distance data transmission, intensive R\&D is now providing us with technologies for high-performance Si-based photonic modules with complex device integrations (Doerr, 2015). In such high-performance applications, various assisting technologies should be implemented on the silicon photonic platform. For example, the resolution and dynamic range of silicon-based interference devices, such as wavelength filters, are considerably limited by fabrication errors in microfabrication processes. To overcome such limitations, additional waveguide systems, based on silicon nitride and silicon-rich silica, have been implemented (Yamada et al., 2014; Doerr, 2015).

Additional waveguide systems can also provide novel functionalities for further performance improvements. For example, the thermo-optic response of photonic devices can be controlled by combining silicon nitride and silicon waveguides, which could guarantee temperature-insensitive operation of data transmission systems (Hiraki et al., 2015). Thermo-optic responses can also be widely controlled by using titania as a cladding material in a Si waveguide (Lee, 2015). Moreover, an additional waveguide system can expand the application field of the Si photonic platform. For instance, silicon nitride waveguides, which are transparent to visible light, can be used to construct compact bio-sensing systems on a small Si chip (Wang et al., 2015).

Light-source integration, which is the most important open issue for the Si photonic platform, requires the help of other materials. For this purpose, III-V semiconductor materials have been bonded on silicon by using various hybrid integration techniques, such as direct die-to-wafer bonding (Fang et al., 2006; Luo et al., 2015). For monolithic integration, Ge-based light sources are now being studied intensively. The most important technology for Ge-based light sources is bandgap engineering, which aims to achieve a direct transition in Ge, which is originally an indirecttransition material. The recent status of Ge-based light sources on $\mathrm{Si}$ is reviewed in this special issue (Saito et al., 2014; Geiger et al., 2015). Mechanical stress and heavily doped n-type carriers 
would significantly contribute to making Ge a direct-transition material. GeSn alloy is also a very attractive material for light sources on a Si platform because Sn, which can easily be dissolved in Ge, offers an important degree of freedom in band-gap engineering (Mosleh et al., 2015). Another approach now being investigated for monolithic integration of light sources is Si-based electro-luminescence at room temperature, although its physical origin has not fully understood ( $\mathrm{Li}$ et al., 2015).

The Si-based photonic platform requires various assisting materials for accomplishing practical photonic functions.

\section{REFERENCES}

Doerr, C. R. (2015). Silicon photonic integration in telecommunications. Front. Phys. 3:37. doi:10.3389/fphy.2015.00037

Fang, A. W., Park, H., Cohen, O., Jones, R., Paniccia, M. J., and Bowers, J. E. (2006). Electrically pumpled hybrid AlGaInAs-silicon evanescent laser. Opt. Express 14, 9203-9216. doi:10.1364/OE.14.009203

Geiger, R., Zabel, T., and Sigg, H. (2015). Group IV direct band gap photonics: methods, challenges, and opportunities. Front. Mater. 2:52. doi:10.3389/ fmats.2015.00052

Hiraki, T., Fukuda, H., Yamada, K., and Yamamoto, T. (2015). Small sensitivity to temperature variations of Si-photonic Mach-Zhender interferometer using Si and SiN waveguides. Front. Mater. 2:26. doi:10.3389/fmats.2015.00026

Lee, J.-M. (2015). Ultrahigh temperature-sensitive silicon MZI with titania cladding. Front. Mater. 2:36. doi:10.3389/fmats.2015.00036

Li, S., Gao, Y., Fan, R., Li, D., and Yang, D. (2015). Room-temperature near-infrared electroluminescence from boron-diffused silicon pn-junction diodes. Front. Mater. 2:8. doi:10.3389/fmats.2015.00008

Luo, X., Cao, Y., Song, J., Hu, X., Cheng, Y., Li, C., et al. (2015). High-throughput multiple dies-to-wafer bonding technology and III/V-on-Si hybrid lasers for heterogeneous integration of optoelectronic integrated circuits. Front. Mater. 2:28. doi:10.3389/fmats.2015.00028

Mosleh, A., Alher, M. A., Cousar, L. C., Du, W., Ghetmiri, S. A., Pham, T., et al. (2015). Direct growth of $\mathrm{Ge}_{1-x} \mathrm{Sn}_{\mathrm{x}}$ films on $\mathrm{Si}$ using a cold-wall ultra-high
Fortunately, it has superior flexibility and robustness for integrating these materials. Along with photonic functions, the Si-based photonic platform can integrate electronic functions monolithically. In other words, we are on the verge of obtaining an ultimate technology that can integrate all photonic and electronic functions on a single Si chip.

\section{ACKNOWLEDGMENTS}

I would like to acknowledge all the authors, reviewers, editors, and publishers, who have supported this Research Topic.

vacuum chemical-vapor-deposition system. Front. Mater. 2:30. doi:10.3389/ fmats.2015.00030

Saito, S., Gardes, F. Y., Al-Attili, A. Z., Tani, K., Oda, K., Suwa, Y., et al. (2014). Group IV light sources to enable the convergence of photonics and electronics. Front. Mater. 1:15. doi:10.3389/fmats.2014.00015

Wang, J., Yao, Z., and Poon, A. W. (2015). Silicon-nitride-based integrated optofluidic biochemical sensors using a coupled-resonator optical waveguide. Front. Mater. 2:34. doi:10.3389/fmats.2015.00034

Yamada, K., Tsuchizawa, T., Nishi, H., Kou, R., Hiraki, T., Takeda, K., et al. (2014). High-performance silicon photonics technology for telecommunications applications. Sci. Technol. Adv. Mater. 15, 024603. doi:10.1088/1468-6996/15/2/024603

Conflict of Interest Statement: The author declares that the research was conducted in the absence of any commercial or financial relationships that could be construed as a potential conflict of interest.

Copyright (c) 2015 Yamada. This is an open-access article distributed under the terms of the Creative Commons Attribution License (CC BY). The use, distribution or reproduction in other forums is permitted, provided the original author(s) or licensor are credited and that the original publication in this journal is cited, in accordance with accepted academic practice. No use, distribution or reproduction is permitted which does not comply with these terms. 